\title{
The influence of massive stars on the interstellar medium
}

\author{
M. Sally Oey \\ Space Telescope Science Institute, \\ 3700 San Martin Drive, Baltimore, MD 21218, USA
}

\begin{abstract}
On scales ranging from pcs to kpcs, the relationship between stellar and gaseous galactic components forms the basis for interpreting observations of galaxies and understanding galaxy formation and evolution. Feedback effects from massive stars dominate the structure, ionization, kinematics, and enrichment of the gaseous ISM in star-forming galaxies. On galactic scales, the ionizing radiation from these stars creates populations of $\mathrm{H}$ II regions and the diffuse, warm ionized medium. Likewise, superbubbles created by stellar winds and supernovae strongly influence the structure, kinematics, and balance of the multiphase ISM. This contribution reviews these feedback effects of massive stars on the global ISM.
\end{abstract}

\section{Introduction}

The progenitors of Wolf-Rayet stars are, for the most part, thought to be the most massive stars in the initial mass function. This population of extremely luminous stars can strongly affect the global properties of the interstellar medium of their host galaxies.

Table 1 summarizes relevant parameters for stars of type $\mathrm{WR}, \mathrm{O} 3 \mathrm{~V}$, and O9V. Over this range of spectral types, the mass-loss rates $\dot{M}$ span 4 orders of magnitude, associated with stellar winds having mechanical luminosities $L$ spanning 3 orders of magnitude. The radii $R$, for adiabatic stellar wind bubbles created by constant $L$ over timescale $\Delta t$, are given in column 6 , assuming a uniform ambient density $n=1 \mathrm{~cm}^{-3}$. The WR bubbles are somewhat smaller than O star bubbles owing to the shorter lifetime of the WR phase in comparison to the H-burning phase. Similarly, the Strömgren radii $R_{\text {str }}$ for the associated spectral types are given in column 8 , using the ionizing photon emission rates $Q\left(\mathrm{H}^{0}\right)$ (column 7) of Schaerer \& de Koter (1997) for O stars, and typical estimate for WR stars by e.g., Crowther \& Smith (1996). Although typically $R_{\text {str }}>R$ in Table 1, I emphasize that this is not necessarily to be expected, considering that $\mathrm{O}$ stars continuously evolve to lower $T_{\text {eff }}$ during the H-burning phase. Also, $R_{\text {str }} \propto n^{-2 / 3}$ while $R \propto n^{-1 / 5}$ (once the SNR has entered its Sedov phase), thus the ambient density will also affect the relationship between the shell and Strömgren radius. So while many shells will be fully ionized, others will not; the point in Table 1 is to demonstrate the considerable spatial influence of these stars both mechanically and by radiation. Finally, the terminal supernovae will eventually dominate the mechanical and element yields.

The influence of this stellar population on the global ISM can determine the phase balance, structure, kinematics, and chemistry of the multi-phase ISM in 
Table 1. Feedback parameters of massive stars.

\begin{tabular}{llcccccc}
\hline type & $\begin{array}{l}M_{\text {ZAMS }} \\
\left(\mathrm{M}_{\odot}\right)\end{array}$ & $\begin{array}{c}\Delta t \\
(\mathrm{yr})\end{array}$ & $\begin{array}{c}-\log \dot{M} \\
\left(\mathrm{M}_{\odot} \mathrm{yr}^{-1}\right)\end{array}$ & $\begin{array}{c}\log L \\
\left(\mathrm{erg} \mathrm{s}^{-1}\right)\end{array}$ & $\begin{array}{c}R \\
(\mathrm{pc})\end{array}$ & $\begin{array}{c}\log Q\left(\mathrm{H}^{0}\right) \\
\left(\mathrm{s}^{-1}\right)\end{array}$ & $\begin{array}{c}R_{\text {str }} \\
(\mathrm{pc})\end{array}$ \\
\hline & & & & & & & \\
WR & $60 ?+$ & $3 \times 10^{5}$ & $4-5$ & 37.6 & 27 & 49 & 140 \\
O3V & $85+$ & $3 \times 10^{6}$ & $5-6$ & 37.0 & 83 & 49.9 & 280 \\
O9V & $20-25$ & $8 \times 10^{6}$ & $7-8$ & 34.4 & 44 & 48.5 & 95 \\
\hline
\end{tabular}

star-forming galaxies. It is possible that the feedback mechanisms from massive stars determine the properties of all of the principal phases of the ISM. The mechanical action resulting from $\mathrm{OB}$ associations and individual supernovae is the likely culprit for the network of shells and filaments that characterize the structure of the cool ISM seen in H I synthesis maps of, for example, the LMC (Dopita et al., these Proceedings), and IC 2574 and Holmberg II (Brinks, these Proceedings). Since the hot $\left(10^{6-7} \mathrm{~K}\right)$, ionized component of the ISM is thought to originate within these superbubbles and supernova remnants (SNRs), the star formation rate and evolution of these shells can determine the balance between the hot ionized medium (HIM) and cooler gas phases. Likewise, the ionizing radiation of massive star populations is responsible for the warm $\left(10^{4} \mathrm{~K}\right)$ ionized medium (WIM) in star-forming galaxies. It is even possible that superbubble action regulates the formation of the coldest, cloud components of the ISM, as the shells sweep up large quantities of gas and merge, perhaps triggering gravitational collapse of molecular and H I clouds. Subsequent star formation would then complete the cycle between the stellar and gaseous components of star-forming galaxies.

\section{Mechanical feedback}

There are many possible evolutionary models for OB shells, depending on the assumed energy loss or conservation in the shell and interior, and ambient environment (see, e.g., Ostriker \& McKee 1988; Koo \& McKee 1992). However, the simplest standard models are still among the most widely used. For a single supernova (SN), representing an isolated, point energy deposition, the SNR evolution can be described by the Sedov (1959) relation:

$$
R \propto\left(\frac{E}{n}\right)^{1 / 5} t^{2 / 5}
$$

where $E$ is the input mechanical energy and $t$ is elapsed time. For a continuous energy injection $\dot{E}$, the analogous shell evolution for an adiabatic, thin shell bubble is (Pikel'ner 1968):

$$
R \propto\left(\frac{\dot{E}}{n}\right)^{1 / 5} t^{3 / 5}
$$


In the case of a stellar wind bubble,

$$
\dot{E}=L=\frac{1}{2} \dot{M} v_{\infty}^{2}
$$

where $v_{\infty}$ is the wind terminal velocity; while for a multi-SN superbubble, $\dot{E}$ may be approximated as (McCray \& Kafatos 1987):

$$
\dot{E}=E_{\text {sn }} \times(\text { SN rate }),
$$

where $E_{\mathrm{sn}}$ is the supernova energy.

With an assumed SN rate and spatial distribution of progenitors, it was realized over two decades ago that these models can predict a 'Three-phase Model', of a Galactic ISM dominated in volume by the HIM (Cox \& Smith 1974; McKee \& Ostriker 1977). This contrasted with the 'Two-phase Model' (Field, Goldsmith, \& Habing 1969) having only a minor contribution from the HIM. Determining the 'porosity' of the Milky Way ISM, essentially the filling factor of the HIM, has therefore been somewhat of a Holy Grail in the study of the Galactic ISM for about two decades.

Clearly, the superbubble action caused by massive star populations plays a critical role here. There are numerous studies that model the ISM, incorporating this feedback in various ways, and varying levels of detail. Many of these are numerical models, including hydrodynamic models such as those initiated by Chiang \& Bregman (1988); the most recent are able to resolve local phenomena such as shock structure (e.g., Thornton et al. 1998). Theoretical models have been reviewed by Mac Low (1999). Detailed numerical models are superior for understanding the consequences of many physical processes such as heating and cooling, radiative transfer, and shell evolution and structure. However, analytic approaches can still yield useful insights (e.g., Heiles 1987, 1990). A recent example is the derivation of the superbubble size distribution by Oey \& Clarke (1997). With simple assumptions for the ambient density and end-stage shell evolution, the adiabatic model described by equation 2 predicts simple analytic functions of the shell size distribution $N(R) d R$ for various combinations of mechanical luminosity function $\phi(L) d L$ and creation rate $\psi(t)$ of OB associations. Figure 1 compares the predicted form of $N(R)$ for the combinations $(i)$ continuous $\psi(t)=\psi_{0}=$ constant, $\phi(L)=L_{0}=$ constant; (ii) single-burst $\psi$, $\phi(L) d L \propto L^{-\beta} d L$; and (iii) continuous $\psi(t)=\psi_{0}, \phi(L) d L \propto L^{-\beta} d L$. These derivations assume constant input power $L$ for all objects, over the lifetime of the lowest-mass stars expected to explode as SNe. It is also assumed that the shell growth stalls at radius $R_{\mathrm{f}}$ and age $t_{\mathrm{f}}$ when the interior pressure decreases to that of the ambient medium. For $N(R) d R \propto R^{-\alpha} d R$, we obtain for case (i) $\alpha=2 / 3$; for case (ii) $\alpha=1-2 \beta, R<R_{\mathrm{f}}\left(t_{\mathrm{b}}\right)$ and $\alpha=4-5 \beta, R>R_{\mathrm{f}}\left(t_{\mathrm{b}}\right)$; and for case (iii) $\alpha=2 / 3, R<R_{\mathrm{f}}\left(L_{\min }\right)$, and $\alpha=1-2 \beta, R>R_{\mathrm{f}}\left(L_{\min }\right)$. $L_{\text {min }}$ is the minimum $L$ in the mechanical luminosity function, thus $R_{\min }$ corresponds to individual SNRs; and $t_{\mathrm{b}}$ is the age for the burst. The $\mathrm{H}_{\mathrm{I}}$ shell catalog of the SMC (Staveley-Smith et al. 1997) yields $\alpha=2.7 \pm 0.6$, in excellent agreement with the predicted value of $2.8 \pm 0.4$ for case ( $i i i)$, using $\beta$ derived from the $\mathrm{H}$ II region luminosity function measured by Kennicutt et al. (1989).

The analytic treatment of the size distribution allows a straightforward derivation of the interstellar porosity parameters $Q_{2 \mathrm{D}}$ (2-dimensional disk) and 


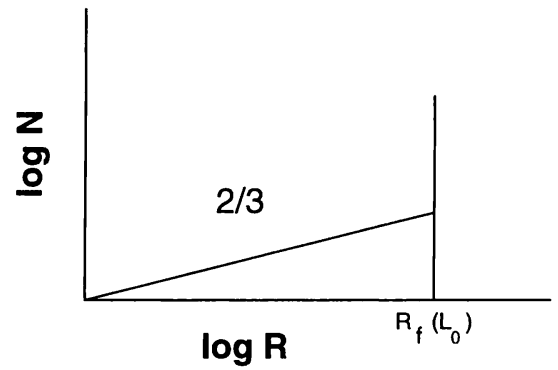

a)

constant $\psi$

single $L$

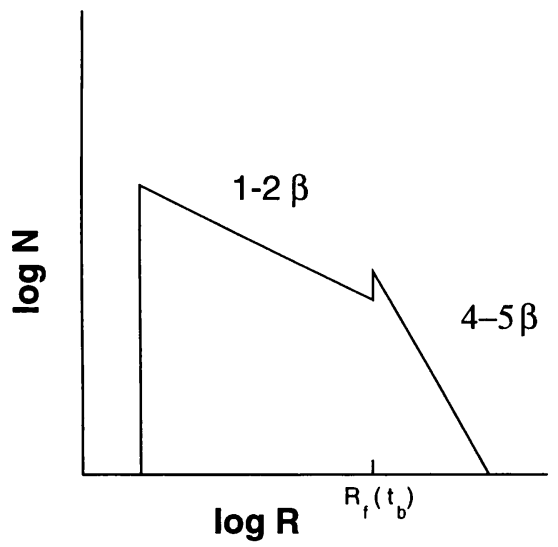

b)

burst $\psi$

$L$ spectrum

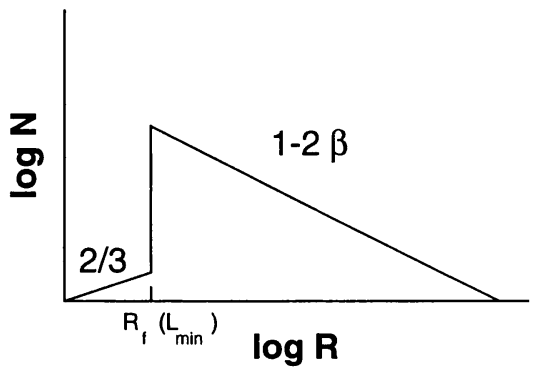

c)

constant $\psi$

$L$ spectrum

Figure 1. Schematic forms of the superbubble size distribution $\log N(\log R)$ for different combinations of creation rate $\psi$ and mechanical luminosity function $\phi(L)$, as described in the text. The predicted dominant slope is shown for each zone. 
$Q_{3 \mathrm{D}}$ (3-dimensional cylinder):

$$
\begin{aligned}
Q_{2 \mathrm{D}} & \propto \frac{L_{e}^{1-\beta}}{4-2 \beta}\left[1-\left(\frac{R_{\min }}{R_{e}}\right)^{4-2 \beta}\right] \\
Q_{3 \mathrm{D}} & \propto \frac{L_{e}^{1-\beta}}{5-2 \beta}\left[1-\left(\frac{R_{\min }}{R_{e}}\right)^{5-2 \beta}\right],
\end{aligned}
$$

where $L_{e}$ and $R_{e}$ are constant, scaling parameters and $R_{\min }=R_{\mathrm{f}}\left(L_{\min }\right)$. For Galactic-like ISM parameters, the derived porosities are substantially $<1$ for three out of the four galaxies we examined, suggesting a low filling factor for the HIM. As a check, the predicted SN rates are also in reasonable agreement with observed rates. However, for the Milky Way, we obtain $Q_{2 \mathrm{D}} \simeq 0.2$ using the $\mathrm{H}$ II region LF, but $Q_{2 \mathrm{D}} \simeq 4$ based on the empirical SN rate. A complete derivation and discussion of the these superbubble size distributions and interstellar porosities can be found in Oey \& Clarke (1997).

\section{Radiative feedback}

The ionizing effect of this same massive star population manifests itself in the resulting warm, ionized medium. This is comprised of two principal components: the familiar, higher-density $\mathrm{H}$ II regions; and the warm $\left(10^{4} \mathrm{~K}\right)$, diffuse, ionized gas that is seen to pervade the ISM in star-forming galaxies. The most notable characteristics of the well-studied nebular population, are its power-law luminosity function (e.g., Kennicutt et al. 1989; Walterbos \& Braun 1992; Banfi et al. 1993); and its exponential size distribution (van den Bergh 1981; Hodge 1983, 1987). The existence of these differing functional forms has yet to be reconciled. Oey \& Clarke (1999) predict variations in the form of the luminosity function caused by statistical effects and luminosity evolution, thus it may be worth reexamining whether the size distribution can be described by a bimodal power law, consistent with predictions and observations of the luminosity function.

The diffuse component of the WIM is also generally thought to be ionized by the massive star population, although this has not been conclusively demonstrated. The Milky Way, a representative star-forming galaxy, has a diffuse WIM component with a scale height of about $1 \mathrm{kpc}$ and filling factor of about 0.2 (e.g., Walterbos 1991). An interesting recent discovery is the identification of apparently different kinematic components by Wang et al. (1997) in a sample of external galaxies. They find a 'disturbed' component showing velocity dispersions of up to $100 \mathrm{~km} \mathrm{~s}^{-1}$, with high [OIII] excitation; and a 'quiescent' component with velocity dispersions of $\lesssim 50 \mathrm{~km} \mathrm{~s}^{-1}$ corresponding to lower [OIII] levels. Intrigueingly, in the edge-on galaxy NGC 891, Rand (1998) finds a correlation between galactic latitude and [OIII] excitation. It is tempting to speculate that this high-latitude, high-excitation gas corresponds to the disturbed component found by Wang et al. in face-on galaxies. A further interesting development is that measurements of [OI $] \lambda 6300 / \mathrm{H} \alpha$ indicate that most of the diffuse $\mathrm{H} \alpha$ in the Galaxy is fully ionized, and not originating from cloud edges (Reynolds et al. 1998).

There are several lines of evidence pointing to massive stars as the ionizing source for the diffuse WIM. One remarkable feature of this gas is the fact 
that it consistently occupies roughly $40 \%$ of the total $\mathrm{H} \alpha$ luminosity of the parent galaxies, independent of star formation rate or Hubble type (Hoopes et al. 1996; Ferguson et al. 1996). This strongly suggests an ionizing source internal to the galaxies, rather than external. The cited studies also find stronger diffuse emission in close proximity to conventional $\mathrm{H}$ II regions, suggesting that these may be leaking Lyman continuum photons; such is indeed found to be the case for many nebulae (Oey \& Kennicutt 1997). The emission-line spectrum of the gas is broadly consistent with photoionization by hot stars (Mathis 1986; Bland-Hawthorn et al. 1997; but see the caveat below). The [SII]/H $\alpha$ ratio increases with distance from $\mathrm{OB}$ associations, in accordance with the ionization parameter becoming increasingly more dilute: [SIr] $/ \mathrm{H} \alpha$ is anticorrelated with galactic latitude (Dettmar \& Schulz 1992; Rand 1997), as expected for ionizing stars concentrated in the disk. In addition, $[\mathrm{SII}] / \mathrm{H} \alpha$ is also anticorrelated with $\mathrm{H} \alpha$ surface brightness (Wang et al. 1998), as expected if the ionizing stars are associated with regions of high $\mathrm{H} \alpha$ surface brightness.

Can the Lyman continuum photons actually travel the necessary hundreds of pc to ionize the diffuse gas? Models by Miller \& Cox (1993) and Dove \& Shull (1994) suggest that it is possible. Also, Ferrara et al. (1996) demonstrate that there may be significant $\mathrm{H} \alpha$ emission from scattered light at up to three times the dust scale height. Empirically, Hunter \& Gallagher (1997) find that filaments at distances as far as a $\mathrm{kpc}$ from the nearest $\mathrm{OB}$ associations still appear to be photoionized.

However, outstanding problems remain with the hypothesis of massive star ionization of the diffuse gas. Emission-line ratios of $\mathrm{He}$ I $\lambda 5876 / \mathrm{H} \alpha$ (Reynolds \& Tufte 1995) and radio recombination lines of $\mathrm{H}$ and $\mathrm{He}$ (Heiles et al. 1996) imply a softer ionizing spectrum than expected from the hot star population. Rand (1998) also finds evidence in the diffuse WIM spectrum for a non-negligible contribution by an additional ionizing source. However, the bulk of evidence remains in favor of massive stars dominating the ionization of this ISM component.

\section{Other feedback effects}

Chemical feedback deserves at least a brief mention; readers are referred to the work of Kobulnicky (these proceedings) for a more thorough discussion. The chemical products of the massive star population are a well-known tracer for star formation history and galaxy evolution, but the details of the mixing processes are at present poorly understood. These must depend on the relationship between star formation and the interstellar processes and properties. The problem is receiving renewed attention in recent years, with respect to mixing within the star forming body of the galaxy (e.g., Roy \& Kunth 1995; Martin \& Belley 1997; Kobulnicky, these Proceedings), and transfer of elements between disk and halo (e.g., Li \& Ikeuchi 1992; Tenorio-Tagle 1996; Silich \& Tenorio-Tagle 1998). Owing to the mechanical feedback effects discussed above, the enrichment of the intergalactic medium is also presumed to originate from the massive stellar population (e.g., Larson \& Dinerstein 1975; David et al. 1990; Gibson 1994).

Finally, the mechanical feedback may be able to trigger renewed star formation, as large quantities of gas are swept up by shells. This possibility has been examined theoretically by several groups, e.g., McCray \& Kafatos (1987); 
Ehlerová et al. (1997); Efremov \& Elmegreen (1998). Observational candidates for superbubble-triggered star formation have also been identified (Walborn \& Parker 1992; Oey \& Massey 1995; Oey \& Smedley 1998).

\section{Conclusion}

It is thus apparent that feedback effects may well drive evolutionary processes in the host galaxies. While Wolf-Rayet stars themselves usually do not dominate feedback phenomena, they are nevertheless an important component, and their progenitors constitute the dominant energy source. The kinetic and radiative effects of the most massive stellar populations strongly affect the structure, phase balance, enrichment, and kinematics of the multi-phase ISM. These in turn can drive star formation, chemical evolution, and galactic structure. Thus, the time-dependence of the feedback processes can be largely responsible for global evolutionary effects in star-forming galaxies. Ultimately, the most massive stars have far-reaching influence that may extend well beyond their host galaxies, to the phase balance and enrichment of the intergalactic medium, and perhaps even beyond.

Acknowledgments. I am grateful for an IAU Travel Grant and the kind last-minute assistance from the organizers that made possible my participation in this meeting.

\section{References}

Banfi, M., Rampazzo, R., Chincarini, G., Henry, R.B.C. 1993, A\&A 280, 373

Bland-Hawthorn, J., Freeman, K.C., Quinn, P.J. 1997, ApJ 490, 143

Chiang, W.-H. Bregman, J.N. 1988, ApJ 328, 427

Chu, Y.-H., Mac Low, M.-M. 1990, ApJ 365, 510

Chu, Y.-H., Chang, H.-W., Su, Y.-L., Mac Low, M.-M. 1995, ApJ 450, 157

Chu, Y.-H., Wakker, B., Mac Low, M.-M., García-Segura, G. 1994, AJ 108, 1696

Cox, D.P., Smith, B.W. 1974, ApJ 189, L105

Crowther, P.A. Smith, L.J. 1996, A\&A 305, 541

David, L.P., Forman, W., Jones, C. 1990, ApJ 359, 29

Dettmar, R.-J., Schulz, H. 1992, A\&A 254, L25

Domgoergen, H., Bomans, D.J., De Boer, K.S. 1995, A\&A 296, 523

Dove, J.B. Shull, J.M. 1994, ApJ 430, 222

Efremov, Y.N., Elmegreen, B.G. 1998, MNRAS 299, 643

Efremov, Y.N., Elmegreen, B.G., Hodge, P.W. 1998, ApJ 501, L163

Ehlerová, S., Palouš, J., Theis, C., Hensler, G. 1997, A\&A 328, 121

Elmegreen, B.G. 1997, ApJ 477, 196

Ferguson, A.M.N., Wyse, R.F.G., Gallagher,J.S., Hunter, D.A. 1996, AJ 111, 2265

Ferrara, A., Bianchi, S., Dettmar, R.-J., Giovanardi, C. 1996, ApJ 467, L69

Field, G.B., Goldsmith, D.W., Habing, H.J. 1969, ApJ 155, L149

Gibson, B.K. 1994, MNRAS 271, 35

Heiles, C. 1987, ApJ 315, 555

Heiles, C. 1990, ApJ 354, 483 
Heiles, C., Koo, B.-C., Levenson, N.A., Reach, W.T. 1996, ApJ 462, 326

Hodge, P.W. 1983, AJ 88, 1323

Hodge, P.W. 1987, PASP 99, 915

Hoopes, C.G., Walterbos, R.A.M., Greenawalt, B.E. 1996, AJ 112, 1429

Kennicutt, R.C., Edgar, B.K., Hodge, P.W. 1989, ApJ 337, 761

Koo, B.-C., McKee, C.F. 1992, ApJ 388, 93

Larson, R.B., Dinerstein, H.L. 1975, PASP 87, 911

Li, F., Ikeuchi, S. 1992, ApJ 390, 405

Mac Low, M.-M. 1999, in: A.R. Taylor, T.L. Landecker \& G. Joncas (eds.), New Perspectives on the Interstellar Medium, ASP-CS 168, ...

Magnier, E.A., Chu, Y.-H., Points, S.D., Hwang, U., Smith, R.C. 1996, ApJ 464, 829

Martin, P., Belley, J. 1997, A\&A 321, 363

Mathis, J.S. 1986, ApJ 301, 423

McCray, R., Kafatos M. 1987, ApJ 317, 190

McKee, C.F., Ostriker, J.P. 1977, ApJ 218, 148

Meaburn, J. 1980, MNRAS 192, 365

Miller, W.W., Cox, D.P. 1993, ApJ 417, 579

Oey, M.S. 1996, ApJ 467, 666

Oey, M.S., Clarke, C.J. 1997, MNRAS 289, 570

Oey, M.S., Clarke, C.J. 1999, in: J. Franco \& A. Carraminana (eds.), Interstellar Turbulence (Cambridge: CUP), in press

Oey, M.S., Kennicutt, R.C. 1997, MNRAS 291, 827

Oey, M.S., Massey, P. 1995, ApJ 452, 210

Oey, M.S., Smedley, S.A. 1998, AJ 116, 1263

Ostriker, J.P., McKee, C.F. 1988, Rev. Mod. Phys 60, 1

Pikel'ner, S.B. 1968, Astrophys. Lett. 2, 97

Rand, R.J. 1997, ApJ 474, 129

Rand, R.J. 1998, ApJ 501, 137

Reynolds, R.J., Hausen, N.R., Tufte, S.L., Haffner, L.M. 1998, ApJ 494, L99

Reynolds, R.J., Tufte, S.L. 1995, ApJ 439, L17

Roy, J.-R., Kunth, D. 1995, A\&A 294, 432

Schaerer, D., de Koter, A. 1997, A\&A 322, 598

Sedov, L.I. 1959, Similarity and Dimensional Methods in Mechanics, (New York: Academic)

Silich, S.A., Tenorio-Tagle, G. 1998, MNRAS 299, 249

Staveley-Smith, L., Sault, R.J., Hatzidimitriou, D., Kesteven, M., McConnell, D. 1997, MNRAS 289, 225

Tenorio-Tagle, G. 1996, AJ 111, 1641

Thornton, K., Gaudlitz, M., Janka, H.-T., Steinmetz, M. 1998, ApJ 500, 95

van den Bergh, S. 1981, AJ 86, 1464

Walborn, N.R., Parker, J.W. 1992, ApJ 399, L87

Walterbos, R.A.M. 1991, in: H. Bloemen (ed.), The Interstellar Disk-Halo Connection in Galaxies, Proc. IAU Symp. No. 144 (Dordrecht: Kluwer), p. 223

Walterbos, R.A.M., Braun, R. 1992, ApJS 92, 625

Wang, J., Heckman, T.M., Lehnert, M.D. 1997, ApJ 491, 114

Wang, J., Heckman, T.M., Lehnert, M.D. 1998, ApJ 509, 93 


\section{Discussion}

Marston: Regarding the importance of WR stars, don't forget these stars had an O star progenitor phase also, which greatly increases their overall effects on the ISM.

Oey: Absolutely. The influence of individual stars includes the combined effect of the $\mathrm{O}$ and WR phases where applicable.

Silich: I would like to mention that you should be careful if you would like to compare Strömgren radius with bubble radius. A high-density shell could trap all the UV photons, and if the characteristic recombination time for the ambient interstellar gas is shorter than the dynamical time, the $\mathrm{H}$ II zone will be confined by the shell.

Oey: I agree completely that this is another factor that would affect the relative radii of the shell and Strömgen sphere.

Dyson: Are the measured ROSAT X-ray fluxes consistent with radiation from adiabatic SNR and/or wind-driven bubbles?

Oey: For 30 Dor, I believe so, see the work by A.D. Wang, 1998. For other LMC superbubbles, some objects show excesses (Chu \& Mac Low 1990; Wang \& Helfand 1991) while others show consistent X-ray luminosities (Chu et al. 1996). The X-ray excess objects are thought to be heated by SNR impacts on the shell walls.

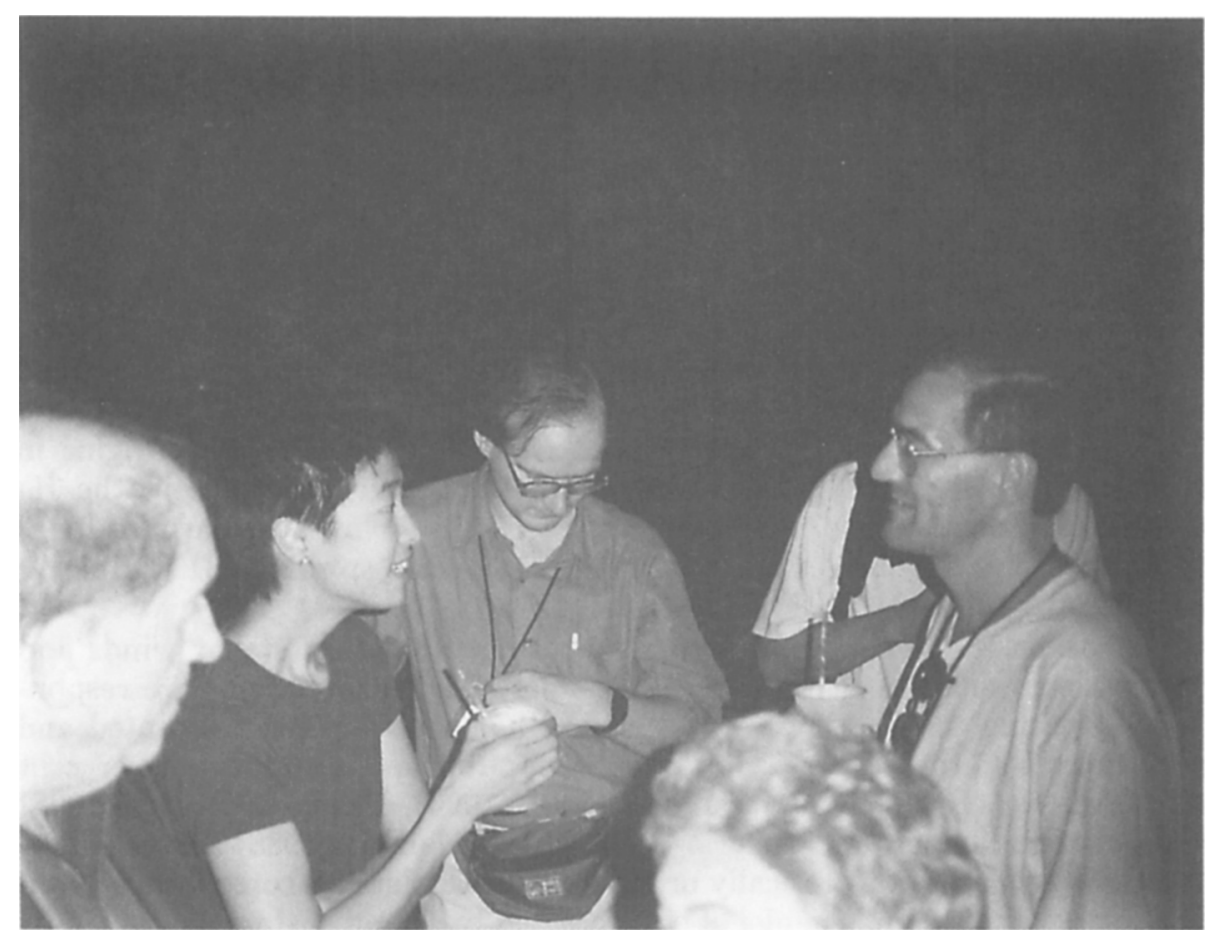

